IMA Journal of Applied Mathematics (2018) Page 1 of 21 doi:10.1093/imamat/xxx000

\title{
Glass sheet redraw through a long heater zone
}

\author{
D. O'KielY*, C. J. W. Breward, I. M. GRIFFiths, P. D. Howell \\ Mathematical Institute, University of Oxford, OX2 6GG UK \\ *okiely@maths.ox.ac.uk \\ AND \\ U. LANGE \\ Schott AG, Hattenbergstrasse 10, 55122 Mainz, Germany
}

[Received on 27 February 2018]

\begin{abstract}
Thin glass sheets may be manufactured using a two-part process in which a sheet is first cast and then subsequently reheated and drawn to a required thickness. The dimensions of the heater zone used for the latter redraw process determine the relative change in the width and thickness of the sheet. When a product with the same cross-sectional aspect ratio as the original sheet is desired, the heater zone through which the sheet is drawn must be long compared with the sheet width. However, deviations from the original aspect ratio due to the finite length of the heater zone can be significant, and the final product is typically thick at the edge compared to the centre. We present a model for redraw of a thin glass sheet and consider the limit in which the heater zone is long compared with the sheet width. We show that the deviations in aspect ratio and thickness depend linearly on the ratio of sheet width to heater zone length and arise due to boundary layers at the sheet ends where the fluid flow adjusts to satisfy imposed boundary conditions. The behaviour inside the boundary layers can be reduced to a canonical problem that is independent of process parameters, and we thus calculate the final thickness and width of the redrawn sheet.
\end{abstract}

Keywords: fluid mechanics; asymptotics; modelling; glass sheet; industrial process

\section{Introduction}

Thin glass sheets are becoming increasingly prevalent in everyday technology, with current applications including substrates in thin-film batteries and thumbprint sensors in smartphones, and with futuristic devices such as bendable televisions and tablets on the horizon (Horvatitsch, 2016; Burke, 2016). Drawing is a technique widely used in the manufacture of these thin glass sheets, as well as that of glass fibres. The most common drawing techniques involve the extrusion of hot molten glass which is stretched as it cools. However, this method requires glass to be formed at appropriate viscosities, and so may be unsuitable when using specialist glasses, which are prone to devitrification or which contain components that will evaporate or react with the atmosphere. This method is also inefficient if only a small quantity of product is required. In such situations, an alternative two-step approach to drawing can be used. A sheet much thicker than the target thickness, known as a preform, is first cast in a mould or by the float-glass process (Howell, 1994), and later fed vertically into a furnace where it is reheated and stretched by the application of a tensile force at a fixed distance downstream. As the glass stretches, it thins and contracts laterally. A schematic of this redrawing process is shown in figure 1. In an ideal scenario, this process would produce long $(\sim 10-100 \mathrm{~m})$, very thin $(\sim 100 \mu \mathrm{m})$ glass sheets of close to uniform thickness, or more generally with a particular desired thickness profile. However, 


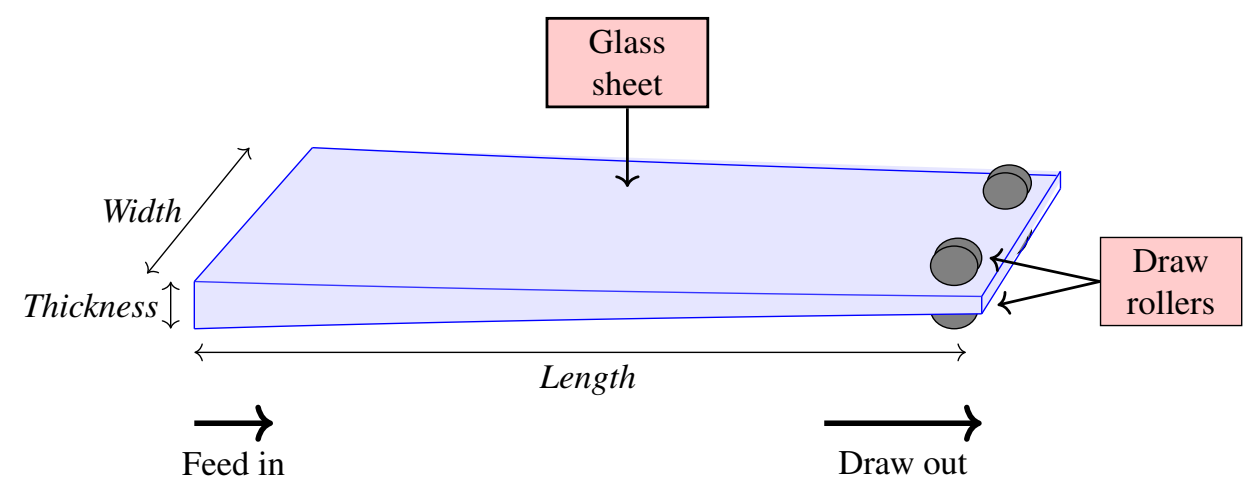

FIG. 1: Schematic of the glass redraw process.

sheets produced in this fashion typically have non-uniform thickness, with the sheet edges thicker than the bulk (see, for example, Filippov \& Zheng, 2010). This non-uniformity is undesirable because it hinders further processing of the sheet as well as causing deterioration in the desired optical properties of the product.

Redraw is an example of an extensional flow problem, in which the in-plane velocity does not vary significantly through the sheet thickness. Studies of extensional flow date back to the experimental work of Trouton (1906), who developed an empirical formula for the stretching of a fibre subject to a tensile force. The Trouton model, which governs the velocity and radius of a fibre under tension, has since been derived, generalized and analysed by various authors. Solutions for steady-state isothermal drawing of a fibre are obtained by Matovich \& Pearson (1969), who consider the effects of viscosity, inertia, surface tension and gravity. Studies typically focus on the leading-order behaviour in the limit of a thin sheet or slender fibre, although Schultz \& Davis (1982) propose an asymptotic expansion in powers of $\varepsilon^{2}$, where $\varepsilon$ is the ratio of fibre radius to its length. Beyond leading order, the boundary conditions at the top and bottom of the fibre cannot be applied directly to the bulk solution, so the authors instead apply a cross-averaged version and thus calculate the proposed $O\left(\varepsilon^{2}\right)$ correction terms.

A model for the viscous flow of a three-dimensional sheet characterized by its small aspect ratio of thickness to other lengthscales is derived by Howell (1994, 1996), and used by Filippov \& Zheng (2010) and O'Kiely et al. (2015) to study the redraw process. Filippov \& Zheng (2010) calculate numerical solutions for the thickness and stress profile in the sheet, and observe that sheet thickness increases towards the edge, and that the thickness profile depends on the temperature profile. O'Kiely et al. (2015) investigate the scenario in which the heater zone is short compared with the sheet width and find that, in this asymptotic limit, the sheet has uniform thickness except in a boundary layer, near the sheet edge, whose extent scales with the heater zone length. The advantage of this regime is that thickness non-uniformities are confined to the neighbourhood of the sheet edge and can thus be removed, while the reduction in sheet width is minimised. The authors thus determine the final thickness profile resulting from an initially flat preform, and also solve the inverse problem to find the initial thickness profile required to redraw a product with uniform thickness. Drawn sheets with thick edges have also been studied in the related problem of casting of polymer films. For example, Dobroth \& Erwin (1986) propose formulas for the bulk and edge thickness in terms of the ratio of the draw to the feed speed, and d'Halewyu et al. (1990) and Silagy et al. (1999) use a thin-sheet model to predict numerically the thickness profile of a cast polymer film. 
In contrast to the short-heater-zone limit considered previously (O'Kiely et al., 2015), in this paper we investigate the limit in which the heater zone is long compared with the sheet width. Howell (1994) shows that, in the absence of surface tension, and in the limit of a long rectangular sheet, each crosssection preserves its aspect ratio as it convects along, so this setup is used in processes where it is desirable to preserve the aspect ratio and cross-sectional shape of the preform. However, in reality it is not economical to construct extremely long heater zones, and the finite length of the heater zone means that the cross-sectional profile of the sheet is not preserved exactly. Indeed, a rectangular preform redraws to a product that is thick at the edges and thin in the centre (see figure 2), and both the width of the product and the thickness variation increase as the ratio of width to heater zone length increases. Furthermore, surface tension acts to pull in the sheet edges, decreasing the final width and increasing the final thickness, and the sharp corners in the preform become rounded (see figure 3). To determine how long a heater zone is required to yield final products acceptably close to rectangular, a clear understanding of the dependence of the final cross-section of the glass sheet on process parameters is required.

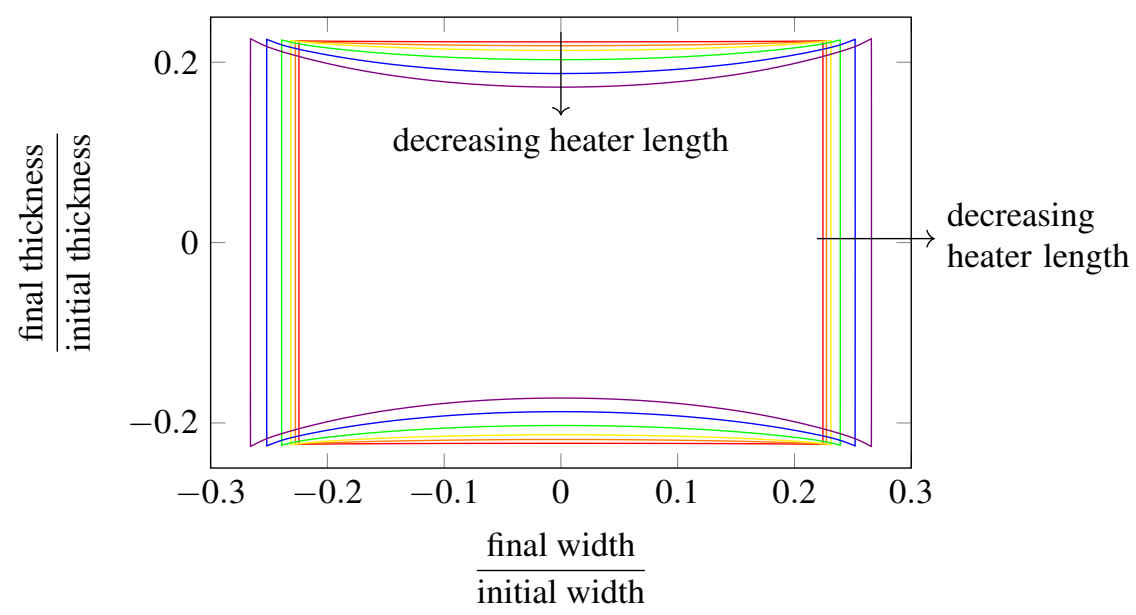

FIG. 2: Final thickness profile of glass after redraw with zero surface tension, with a ratio of preform half-width to heater zone length equal to 0.01 (red), 0.05 (orange), 0.1 (yellow), 0.2 (green), 0.35 (blue) and 0.5 (violet). These are numerical solutions to the three-dimensional Stokes equations calculated in Polyflow (details in $\S 6.2$ ).

In $\S 2$ we present a model for redraw of a thin viscous sheet. We consider the limit where the heater zone is long compared to the preform width in $\S 3$. At leading order in the small aspect ratio of halfwidth to heater length, we recover the result of Howell (1994) that the sheet cross-section is preserved at leading order in the absence of surface tension, and we calculate the change in thickness and width due to surface tension. In $\S 4$ we proceed to next order in the asymptotic expansion to determine the first correction to the leading-order solution, quantifying the deviations from the preform shape when the heater zone has finite length. Matching between the bulk and boundary layers at the sheet ends motivates an expansion in powers of the ratio of heater zone length to preform half-width, $\delta$, as opposed to the $\delta^{2}$ expansion employed in Schultz \& Davis (1982). We thus show that corrections to the leadingorder behaviour are larger than previously predicted, and that simply averaging the boundary conditions at the sheet ends and applying them to the bulk is not appropriate beyond leading order. In $\S 5$ we study 


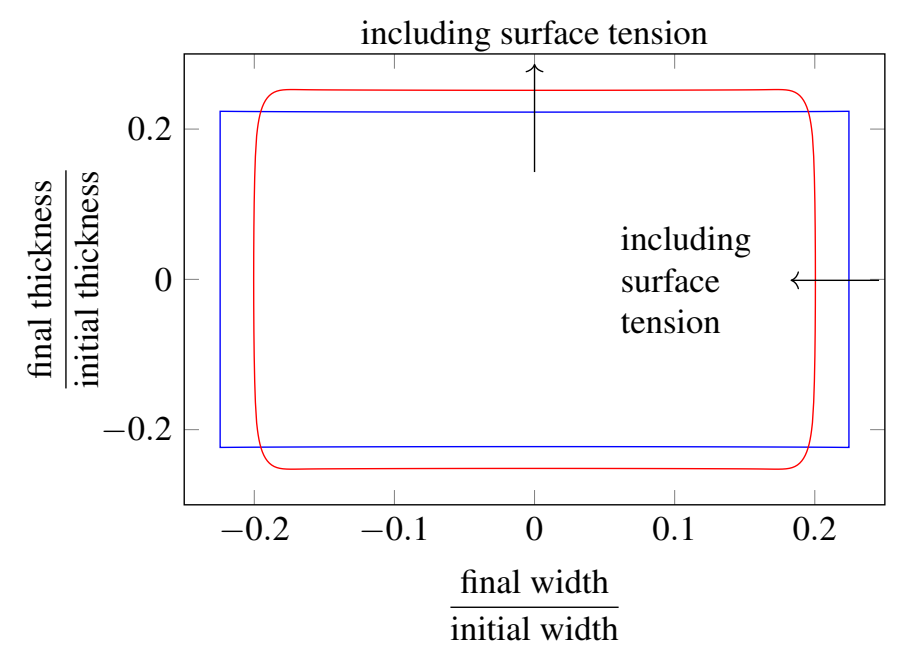

FIG. 3: Final thickness profile of glass after redraw with and without surface tension, with a ratio of preform half-width to heater zone length equal to 0.01 , calculated using Polyflow (details in $\S 6.2$ ).

the behaviour in the boundary layers at either end of the sheet and determine the matching conditions for the bulk problem. The solution of an elliptic boundary-value problem provides the appropriate effective boundary conditions needed to close the bulk problem. In $\S 6$, we use the computed solution to investigate the dependence of the redrawn sheet properties on process parameters. We compare our predictions with numerical solutions to the full three-dimensional problem, and also address the inverse problem: what preform shape redraws to a rectangular final product?

\section{Mathematical model}

We use Cartesian coordinates $\boldsymbol{x}=(x, y, z)$, with the $x$-axis aligned with the direction of drawing, the $y$ axis spanning the width of the sheet as it enters the heater zone, and $z$ in the transverse direction as shown in figure 4. We consider a planar sheet of glass undergoing redraw. In industrial processes of interest, the Reynolds number is small, and we neglect gravity by assuming that the Stokes number $\mathrm{St}=\rho g L^{2} / \mu U$ is small, where $\rho$ and $\mu$ are the gravity and viscosity of the sheet, $g=9.81 \mathrm{~ms}^{-2}$ and $L$ and $U$ are characteristic length and velocity scales respectively. The glass viscosity may vary significantly along the heater zone, but we consider here the case of uniform viscosity and focus on the fundamental scalings underlying the final sheet shape. In principle the sheet motion is governed by the Stokes equations, but we will exploit the slender geometry of the sheet to simplify the problem. We define two aspect ratios

$$
\begin{gathered}
\varepsilon=\frac{\text { preform thickness }}{\text { preform width }}, \\
\delta=\frac{\text { preform half-width }}{\text { length of heater zone }} .
\end{gathered}
$$

In manufacture of glass sheets, the sheet thickness is invariably much smaller than either the length or the width, and it follows that $\varepsilon \ll 1$. We will further focus on scenarios where the heater zone is long compared to the sheet width, and therefore we assume that $\varepsilon \ll \delta \ll 1$. 


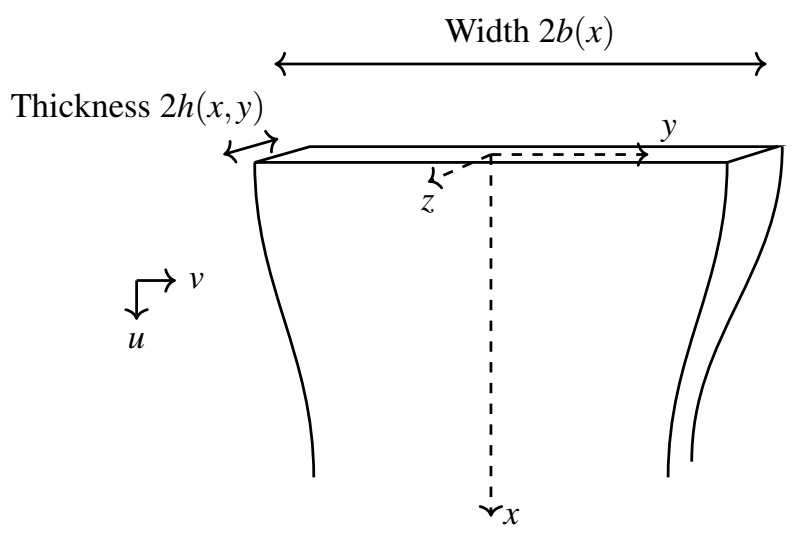

FIG. 4: Three-dimensional glass sheet with width $2 b(x)$ and thickness $2 h(x, y)$ undergoing redraw in the $x$-direction.

In the limit $\varepsilon \rightarrow 0$, the dimensionless in-plane velocity $\boldsymbol{u}=(u, v)$, half-thickness $h$ and half-width $b$ of the sheet are governed by the thin-sheet equations (Howell, 1994). The leading-order in-plane velocities are uniform through the sheet thickness, i.e.,

$$
u=u(x, y), \quad v=v(x, y)
$$

To leading order (in $\varepsilon$ ), the dimensionless net conservation of mass and momentum are given by

$$
\begin{gathered}
(u h)_{x}+(v h)_{y}=0 \\
\delta^{2}\left(4 h u_{x}+2 h v_{y}\right)_{x}+\left(h u_{y}+\delta^{2} h v_{x}\right)_{y}=0 \\
\left(h u_{y}+\delta^{2} h v_{x}\right)_{x}+\left(2 h u_{x}+4 h v_{y}\right)_{y}=0
\end{gathered}
$$

in the domain $(x, y) \in[0,1] \times[-b(x), b(x)]$, where we have scaled $x$ with the heater zone length and $y$ with the initial sheet half-width, we have scaled $u$ and $v$ with the inlet speed $U$ and $\delta U$ respectively, and we have scaled $h$ with the average initial thickness. The sheet shape and speed are known at the top of the heater zone, and the speed at the bottom of the zone is controlled by draw rollers, so we impose the boundary conditions

$$
\begin{gathered}
u=b=1, \quad h=1+\delta \eta_{\text {in }}(y), \quad v=0 \quad \text { on } \quad x=0, \\
u=D, \quad v=0 \quad \text { on } \quad x=1 .
\end{gathered}
$$

We assume here that the solid preform entering at the top and the solid product being removed by rollers at the bottom may be modelled as plug flow. In (2.4a), $\eta_{\text {in }}$ represents a small perturbation to the initial preform shape, with the most common situation of a uniform preform corresponding to $\eta_{\text {in }} \equiv 0$. We will show in $\S 6.3$ how $\eta_{\text {in }}$ may be adjusted to control the final thickness profile of the sheet produced at $x=1$. We assume that $\eta_{\text {in }}(y)$ is symmetric about $y=0$. Without loss of generality, we can then choose the scaling for $h$ such that

$$
\int_{0}^{1} \eta_{\text {in }}(y) \mathrm{d} y=0 .
$$


We shall see below that applying the end conditions (2.4) is not straightforward in the limit $\delta \rightarrow 0$, when the bulk velocity cannot fully satisfy the boundary conditions. The velocity adjusts rapidly in boundary layers at either end of the sheet from the profile imposed by the feed and draw rollers to the bulk profile. At leading order, we may simply impose the feed speed $u(0, y)=1$ and draw speed $u(1, y)=D$ and disregard the transverse velocity conditions. However, the boundary-layer behaviour must be properly resolved for accurate determination of higher-order corrections; we return to this in $\S 5$.

We expect the sheet to be symmetric about $y=0$, i.e.,

$$
v=u_{y}=0 \quad \text { on } \quad y=0 .
$$

Net conservation of mass and momentum at the edge of the sheet lead to the boundary conditions

$$
\begin{gathered}
v=u b^{\prime}(x), \quad \text { on } \quad y=b(x), \\
\left(\begin{array}{cc}
4 h u_{x}+2 h v_{y}+\Gamma & \delta^{-1} h u_{y}+\delta h v_{x} \\
\delta^{-1} h u_{y}+\delta h v_{x} & 2 h u_{x}+4 h v_{y}+\Gamma
\end{array}\right)\left(\begin{array}{c}
-\delta b^{\prime}(x) \\
1
\end{array}\right)=0 \quad \text { on } \quad y=b(x),
\end{gathered}
$$

where

$$
\Gamma=\frac{1}{\varepsilon \delta \mathrm{Ca}}=\frac{\text { heater length }}{\text { preform half-width }} \frac{\gamma}{\mu U}
$$

measures the importance of surface tension relative to viscous effects, with

$$
\mathrm{Ca}=\frac{\mu U}{\gamma}
$$

the capillary number, $\mu$ the viscosity, $U$ the feed speed and $\gamma$ the surface-tension coefficient. We will investigate the regime in which $\Gamma=O(1)$ as $\delta \rightarrow 0$ so that surface tension plays a role at leading order.

\section{Leading-order behaviour}

We examine the behaviour for a very long heater zone by considering the limit $\delta \rightarrow 0$ and expanding variables in powers of $\delta$. Evaluating the $x$-momentum equation (2.3b) at leading order and integrating using the symmetry condition (2.6), we find that the leading-order velocity varies only along the length of the sheet, i.e.,

$$
u_{0}=u_{0}(x),
$$

and the flow is extensional. Evaluating the $y$-momentum equation (2.3c) at leading order and integrating using the free-boundary condition $(2.7 \mathrm{~b})$ we find

$$
2 h_{0} u_{0 x}+4 h_{0} v_{0 y}=-\Gamma,
$$

which represents a net lateral stress balance. Integrating the mass conservation equation (2.3a) across the sheet width and using symmetry condition (2.6), no-flux condition (2.7a) and inlet conditions (2.4a) yields

$$
u_{0} \int_{0}^{b_{0}} h_{0} \mathrm{~d} y=1,
$$

implying a constant net flux along the sheet. Integrating force balance (2.3b) across the sheet width and using no-stress condition (2.7b) yields a cross-averaged longitudinal force balance, which may be simplified using (3.2) to give

$$
\frac{\mathrm{d}}{\mathrm{d} x}\left(\int_{0}^{b_{0}} 3 h_{0} u_{0 x} \mathrm{~d} y+\frac{\Gamma b_{0}}{2}\right)=0
$$


The term in brackets is the net axial tension in the sheet, including viscous and capillary contributions. We note that the Trouton ratio in this regime is 3 ; this is to be expected since in the limit of a long heater zone the sheet is quasi one-dimensional. We obtain a third equation for $u_{0}, h_{0}, b_{0}$ by applying the no-flux condition (2.7a) at leading order and simplifying using (3.2) to obtain

$$
u_{0} b_{0 x}+\frac{1}{2} u_{0 x} b_{0}+\frac{\Gamma}{4} \int_{0}^{b_{0}} \frac{1}{h_{0}} \mathrm{~d} y=0 .
$$

Since we assume that the initial thickness profile is approximately uniform, i.e., $h(0, y)=1+O(\delta)$, it can be shown (see, for example, Howell, 1994) that $h_{0}=h_{0}(x)$, so that the leading-order governing equations (3.3)-(3.5) reduce to

$$
\begin{gathered}
u_{0} b_{0} h_{0}=1, \\
\frac{\mathrm{d}}{\mathrm{d} x}\left(\frac{3}{u_{0}} \frac{\mathrm{d} u_{0}}{\mathrm{~d} x}+\frac{\Gamma b_{0}}{2}\right)=0, \\
\frac{1}{b_{0}} \frac{\mathrm{d} b_{0}}{\mathrm{~d} x}+\frac{1}{2 u_{0}} \frac{\mathrm{d} u_{0}}{\mathrm{~d} x}=-\frac{\Gamma}{4} b_{0},
\end{gathered}
$$

with boundary conditions

$$
u_{0}(0)=h_{0}(0)=b_{0}(0)=1, \quad u_{0}(1)=D .
$$

This leading-order problem has solution

$$
\begin{gathered}
u_{0}(x)=\frac{3 \kappa}{\Gamma+3 \kappa-\Gamma \mathrm{e}^{-\kappa x / 2}} \mathrm{e}^{\kappa x} \\
h_{0}(x)=\left(\frac{\Gamma+3 \kappa-\Gamma \mathrm{e}^{-\kappa x / 2}}{3 \kappa}\right)^{2} \mathrm{e}^{-\kappa x / 2}=\frac{\mathrm{e}^{3 \kappa x / 2}}{u_{0}(x)^{2}} \\
b_{0}(x)=\frac{3 \kappa}{\Gamma+3 \kappa-\Gamma \mathrm{e}^{-\kappa x / 2}} \mathrm{e}^{-\kappa x / 2}=u_{0}(x) \mathrm{e}^{-3 \kappa x / 2},
\end{gathered}
$$

where $\kappa$ satisfies the transcendental equation

$$
\Gamma=\frac{3 \kappa\left(\mathrm{e}^{\kappa}-D\right)}{D\left(1-\mathrm{e}^{-\kappa / 2}\right)} .
$$

We note that $\kappa \geqslant \log (D)$ and $\kappa \rightarrow \log (D)$ as $\Gamma \rightarrow 0$.

The thickness and width profiles of a sheet redrawn with $D=20$ are shown in figure 5 for $\Gamma=0$ and $\Gamma=5$. We observe that surface tension promotes necking-in of the sheet, so that the thickness is larger and the width smaller compared to their values in the absence of surface tension. Using (3.8), we observe that the final thickness $h_{0}(1)$ and width $b_{0}(1)$ of a redrawn sheet with surface tension $\Gamma \neq 0$, compared to their values when $\Gamma=0$, can be quantified by

$$
\frac{h_{0}(1)}{D^{-1 / 2}}=\left(\frac{b_{0}(1)}{D^{-1 / 2}}\right)^{-1}=\mathrm{e}^{3(\kappa-\log (D)) / 2} .
$$

The ratio $h_{0}(1) / D^{-1 / 2}$ is plotted in figure 6 as a function of the surface tension parameter $\Gamma$ for a range of values of the draw ratio $D$. In the limit of negligible surface tension, with $\Gamma \rightarrow 0$ and $\kappa \rightarrow \log (D)$, we see that both $h_{0}(1)$ and $b_{0}(1)$ scale with $D^{-1 / 2}$ and the aspect ratio of the sheet is preserved, as expected. Increasing surface tension leads to lateral contraction of the sheet, accompanied by a transverse thickening by the same factor (namely the right-hand side of equation (3.10)). 


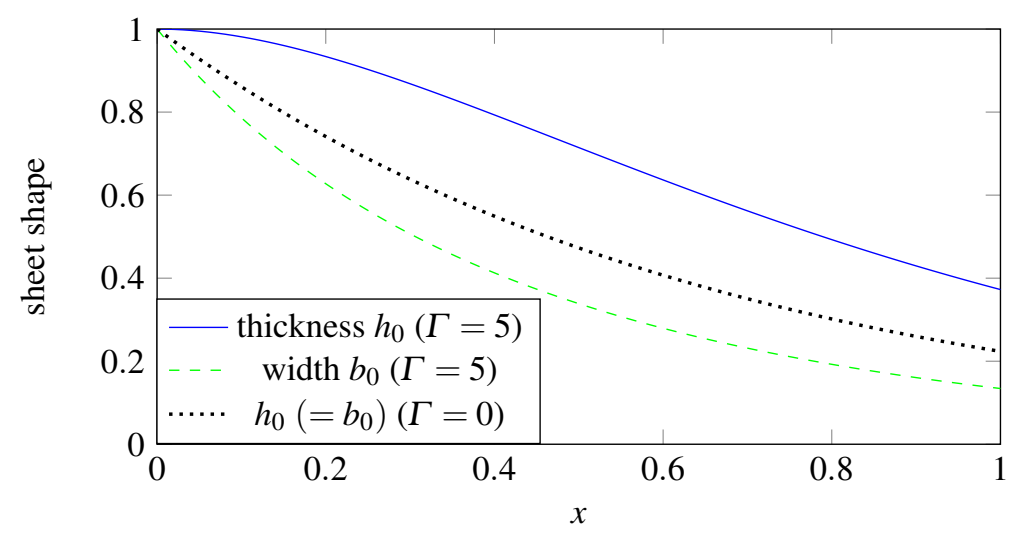

FIG. 5: Leading-order thickness $h_{0}(x)$ and width $b_{0}(x)$ (given by (3.8)) at draw ratio $D=20$ with $\Gamma=5$, compared with $\Gamma=0$ case (dotted line).

\section{Correction terms}

So far, we have calculated the leading-order behaviour of a glass sheet whose initial thickness is approximately uniform (up to perturbations of size $\delta$ ). In this scenario, the leading-order thickness is uniform across the sheet width throughout the redraw process. To describe non-trivial thickness variations and thus quantify the phenomenon of edge thicknening, we now calculate $O(\delta)$ corrections to the leadingorder behaviour. We return to the $x$-momentum equation (2.3b), integrating and applying (2.6) to obtain $u_{1}=u_{1}(x)$, so that the flow is extensional up to $O(\delta)$. Evaluating the $y$-momentum equation (2.3c) at $O(\delta)$ and integrating, we obtain

$$
2 h_{0} v_{1 y}+2 h_{1} v_{0 y}=-h_{0} u_{1 x}-h_{1} u_{0 x},
$$

so that substituting in (3.2) and solving for $v_{1}$ yields

$$
v_{1}=-\frac{y}{2} u_{1 x}+\frac{\Gamma}{4} \frac{1}{h_{0}^{2}} \int_{0}^{y} h_{1} \mathrm{~d} y .
$$

Integrating the mass equation (2.3a) across the sheet width and evaluating at $O(\delta)$ yields

$$
u_{0} \int_{0}^{b_{0}} h_{1} \mathrm{~d} y=-h_{0} b_{0} u_{1}-u_{0} h_{0} b_{1}
$$

We again integrate the $x$-momentum (2.3b) equation across the sheet width, and use (4.3) to eliminate $h_{1}$ to obtain

$$
\frac{\mathrm{d}^{2}}{\mathrm{~d} x^{2}}\left(3 \frac{u_{1}}{u_{0}}\right)=-\frac{\Gamma}{2} \frac{\mathrm{d} b_{1}}{\mathrm{~d} x}
$$

Meanwhile, the no-flux boundary condition (2.7a) together with (4.2)-(4.3) and leading-order solutions (3.8) yields

$$
\frac{\mathrm{d}}{\mathrm{d} x}\left(\frac{b_{1}}{b_{0}}+\frac{u_{1}}{2 u_{0}}\right)+\frac{\Gamma}{4} b_{1}=0 .
$$




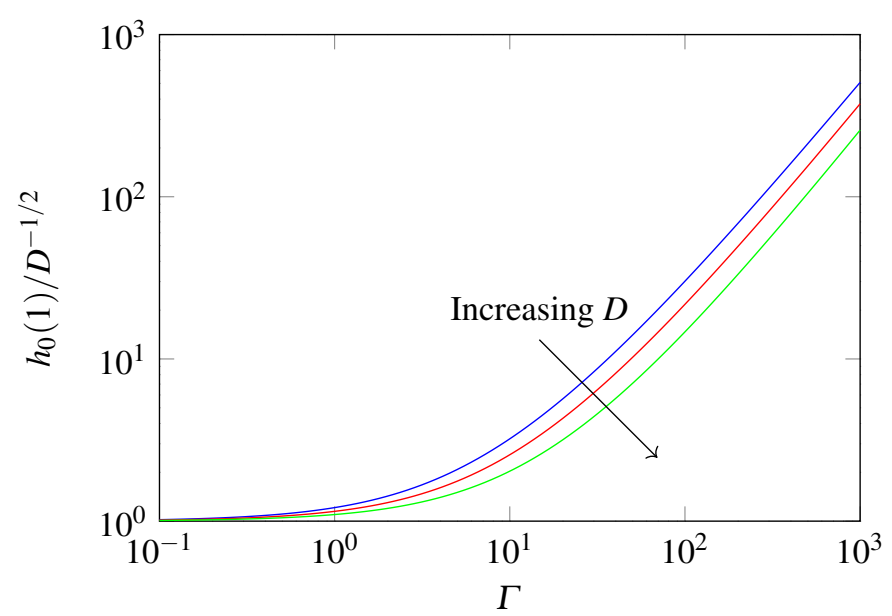

FIG. 6: Final thickness as a function of the surface tension parameter $\Gamma$, compared to the value in the absence of surface tension, for $D=2$ (blue), $D=10$ (red) and $D=100$ (green). The final width and thickness are related by (3.10).

The mass equation (2.3a) also yields

$$
\frac{1}{2} \frac{u_{0 x}}{u_{0}} h_{1}+h_{1 x}-\left(\frac{1}{2} \frac{u_{0 x}}{u_{0}}+\frac{\Gamma}{4} \frac{1}{u_{0} h_{0}}\right) y h_{1 y}=-\frac{1}{2} \frac{u_{1 x}}{u_{0}} h_{0}-\frac{u_{1}}{u_{0}} h_{0 x} .
$$

We can learn more about the thickness correction $h_{1}$ by introducing the scaled $y$-variable $\xi=y / b_{0}(x)$. If $h_{1}$ is now expressed as a function of $x$ and $\xi$, the mass equation (4.6) is transformed to

$$
\frac{\partial}{\partial x}\left(h_{1} \sqrt{u_{0}}\right)=-\sqrt{\frac{u_{1}}{u_{0}}} \frac{\mathrm{d}}{\mathrm{d} x}\left(h_{0} \sqrt{u_{1}}\right) .
$$

The right-hand side is a function of $x$ only, and we deduce that $h_{1}$ is of the form

$$
h_{1}\left(x, \frac{y}{b_{0}}\right)=A(x)+\frac{1}{\sqrt{u_{0}(x)}} g\left(\frac{y}{b_{0}(x)}\right),
$$

where $g$ is an arbitrary integration function. To specify the the decomposition (4.8) uniquely, we set

$$
\int_{0}^{1} h_{1}(x, \xi) \mathrm{d} \xi=A(x), \quad \int_{0}^{1} g(\xi) d \xi=0 .
$$

The governing equations (4.3)-(4.5) can then be solved for

$$
\begin{gathered}
\frac{u_{1}(x)}{u_{0}(x)}=2 u_{1}(0)+A(0)-2 \kappa d_{1} x+\Gamma d_{1} \alpha(x)-\mathrm{e}^{-\kappa x} u_{0}(x)\left[u_{1}(0)+A(0)\right], \\
\frac{b_{1}(x)}{b_{0}(x)}=\kappa d_{1} x+\Gamma d_{1} \alpha(x)-\mathrm{e}^{-\kappa x} u_{0}(x)\left[u_{1}(0)+A(0)\right] \\
\frac{A(x)}{h_{0}(x)}=-2 u_{1}(0)-A(0)+\kappa d_{1} x-2 \Gamma d_{1} \alpha(x)+2 \mathrm{e}^{-\kappa x} u_{0}(x)\left[u_{1}(0)+A(0)\right],
\end{gathered}
$$


where

$$
\alpha(x)=\left(\frac{2\left(\mathrm{e}^{-\kappa x / 2}-1\right)+\kappa x \mathrm{e}^{-\kappa x / 2}}{\Gamma+3 \kappa-\Gamma \mathrm{e}^{-\kappa x / 2}}\right) .
$$

The constants $d_{1}, A(0)$ and $u_{1}(0)$, together with the function $g(\xi)$, must be determined by applying appropriate boundary conditions on $h_{1}, u_{1}$ and $b_{1}$ (given by (4.8) and (4.10)). However, these correction terms do not satisfy all of the boundary conditions given in (2.4); there are boundary layers at either end of the sheet where the solution adjusts from its bulk behaviour to that at the boundaries.

\section{Boundary-layer behaviour}

\subsection{Top boundary layer}

We first consider the boundary layer at the top of the sheet $x=0$ by rescaling $(x, y)=(\delta \hat{x}, \hat{y})$. By expanding the outer solution obtained in $\S \S 3-4$ in term of inner variables, we obtain the matching conditions

$$
\begin{aligned}
& u \sim 1+\delta\left[u_{1}(0)+\left(\kappa-\frac{\Gamma}{6}\right) \hat{x}\right]+\cdots, \\
& v \sim-\left(\kappa+\frac{\Gamma}{3}\right) \frac{\hat{y}}{2}+\cdots, \\
& h \sim 1+\delta\left[A(0)+g(\hat{y})-\left(\frac{\kappa}{2}-\frac{\Gamma}{3}\right) \hat{x}\right]+\cdots, \\
& b \sim 1-\delta\left[u_{1}(0)+A(0)+\left(\frac{\kappa}{2}+\frac{\Gamma}{6}\right) \hat{x}\right]+\cdots,
\end{aligned}
$$

to be applied as $\hat{x} \rightarrow \infty$. This specified far-field behaviour prompts us to seek inner solutions in the form

$$
\begin{aligned}
& u=1+\delta\left[\left(\kappa-\frac{\Gamma}{6}\right) \hat{x}+\left(\kappa+\frac{\Gamma}{3}\right) \hat{u}_{1}(\hat{x}, \hat{y})\right]+O\left(\delta^{2}\right), \\
& v=\left(\kappa+\frac{\Gamma}{3}\right)\left[-\frac{\hat{y}}{2}+\hat{v}_{0}(\hat{x}, \hat{y})\right]+O(\delta), \\
& h=1+\delta\left[\eta_{\text {in }}(\hat{y})-\left(\frac{\kappa}{2}-\frac{\Gamma}{3}\right) \hat{x}+\left(\kappa+\frac{\Gamma}{3}\right) \hat{h}_{1}(\hat{x}, \hat{y})\right]+O\left(\delta^{2}\right), \\
& \hat{b}=1+\delta\left[-\left(\frac{\kappa}{2}+\frac{\Gamma}{6}\right) \hat{x}+\left(\kappa+\frac{\Gamma}{3}\right) \hat{b}_{1}(\hat{x})\right]+O\left(\delta^{2}\right) .
\end{aligned}
$$

Substituting the ansatz (5.2) into (2.3) we find the leading-order governing equations in the boundary layer:

$$
\begin{gathered}
\left(4 \hat{u}_{1 \hat{x}}+2 \hat{v}_{0 \hat{y}}\right)_{\hat{x}}+\left(\hat{u}_{1 \hat{y}}+\hat{v}_{0 \hat{x}}\right)_{\hat{y}}=0, \\
\left(\hat{u}_{1 \hat{y}}+\hat{v}_{0 \hat{x}}\right)_{\hat{x}}+\left(2 \hat{u}_{1 \hat{x}}+4 \hat{v}_{0 \hat{y}}\right)_{\hat{y}}=0, \\
\hat{u}_{1 \hat{x}}+\hat{h}_{1 \hat{x}}+\hat{v}_{0 \hat{y}}=0 .
\end{gathered}
$$


The symmetry conditions (2.6) become

$$
\hat{v}_{0}=\hat{u}_{1 \hat{y}}=0 \quad \text { on } \quad \hat{y}=0,
$$

and the free boundary conditions $(2.7)$ (now at $\hat{y}=1$ ) are transformed to

$$
\begin{array}{r}
\hat{u}_{1 y}+\hat{v}_{0 \hat{x}}=0, \quad 2 \hat{u}_{1 \hat{x}}+4 \hat{v}_{0 \hat{y}}=0 \quad \text { on } \quad \hat{y}=1, \\
\hat{v}_{0}=b_{1 \hat{x}} \quad \text { on } \quad \hat{y}=1,
\end{array}
$$

while the feed conditions (2.4a) become

$$
\begin{array}{r}
\hat{u}_{1}=0, \quad \hat{v}_{0}=\frac{\hat{y}}{2} \quad \text { on } \quad \hat{x}=0, \\
\hat{h}_{1}=\hat{b}_{1}=0 \quad \text { on } \quad \hat{x}=0 .
\end{array}
$$

To satisfy the matching conditions (5.1), we require the normalised variables to have the far-field behaviour

$$
\begin{array}{r}
\hat{u}_{1} \rightarrow c_{1}, \quad \hat{v}_{0} \sim 0 \quad \text { as } \quad \hat{x} \rightarrow \infty, \\
\hat{h}_{1} \rightarrow f(\hat{y})-c_{1}-c_{2}, \quad \hat{b}_{1} \rightarrow c_{2} \quad \text { as } \quad \hat{x} \rightarrow \infty,
\end{array}
$$

for some constants $c_{1}, c_{2}$ and some function $f$.

The recipe for solving the boundary-layer problem is as follows. The velocity components $\hat{u}_{1}$ and $\hat{v}_{0}$ satisfy the linear elliptic system $(5.3 a)-(5.3 b)$ on the fixed domain $[0, \infty) \times[0,1]$, together with boundary conditions (5.5a), (5.6a) and (5.7a). We solve the system numerically using finite-element software FEniCS; since the problem is parameter free it only needs to be solved once. A convergence check shows that the solution may be determined to three decimal places with a $1000 \times 400$ mesh and the domain truncated at $\hat{x}=5$. The resulting boundary-layer solutions for $\hat{u}_{1}$ and $\hat{v}_{0}$ are plotted in figure 7(a) and (b), and the integration constant $c_{1}$ is also evaluated as part of the solution, namely

$$
c_{1}=-0.064 \text {. }
$$

Having solved for $\hat{u}_{1}$ and $\hat{v}_{0}$, we can subsequently determine the thickness and width perturbations, $\hat{h}_{1}$ and $\hat{b}_{1}$, by integrating the mass conservation equation $(5.3 \mathrm{c})$ and kinematic boundary condition $(5.5 \mathrm{~b})$ with respect to $\hat{x}$ :

$$
\begin{gathered}
\hat{h}_{1}(\hat{x}, \hat{y})=-\hat{u}_{1}(\hat{x}, \hat{y})-\int_{0}^{\hat{x}} \hat{v}_{0 \hat{y}}(s, \hat{y}) \mathrm{d} s \\
\hat{b}_{1}(\hat{x})=\int_{0}^{\hat{x}} \hat{v}_{0}(s, 1) \mathrm{d} s=-\int_{0}^{1}\left(\hat{u}_{1}(\hat{x}, \hat{y})+\hat{h}_{1}(\hat{x}, \hat{y})\right) \mathrm{d} \hat{y} .
\end{gathered}
$$

The resulting solutions plotted in figure 7(c) and (d) reveal some interesting behaviour. For small values of $\hat{x}$, the sheet initially thins most rapidly at the edges, so the maximum thickness occurs on the symmetry line $\hat{y}=0$, before evolving towards a far-field profile with the anticipated thick edges. Meanwhile, the width perturbation $\hat{b}_{1}(\hat{x})$ over-shoots somewhat before converging to a constant farfield value: behaviour reminiscent of die swell (see, for example, Silliman \& Scriven, 1980).

By comparing the numerical solutions with the anticipated far-field behaviour (5.7b), we determine the remaining integration constant

$$
c_{2}=\int_{0}^{\infty} \hat{v}_{0}(\hat{x}, 1) \mathrm{d} \hat{x}=0.129
$$


along with the function

$$
f(\hat{y})=c_{2}-\int_{0}^{\infty} \hat{v}_{0 \hat{y}}(\hat{x}, \hat{y}) \mathrm{d} \hat{x}
$$

which is plotted in figure 8 .

Finally, by applying the matching conditions (5.1), we can evaluate the integration constants needed for the first-order outer solution, namely

$$
\begin{aligned}
& u_{1}(0)=\left(\kappa+\frac{\Gamma}{3}\right) c_{1}, \\
& A(0)=-\left(\kappa+\frac{\Gamma}{3}\right)\left(c_{1}+c_{2}\right), \\
& g(y)=\eta_{\text {in }}(y)+\left(\kappa+\frac{\Gamma}{3}\right) f(y) .
\end{aligned}
$$

In particular, we see how the function $g$ governing the thickness variations in the outer solution is given by a combination of the input thickness perturbation $\eta_{\text {in }}$ and the numerically determined function $f$. To determine the remaining constant $d_{1}$, we must now match with the boundary layer at the bottom of the sheet $x=1$.

\subsection{Bottom boundary layer}

We express the thickness and width at the bottom of the sheet in the form

$$
h(1, y)=D^{-2} \mathrm{e}^{3 \kappa / 2}+\delta \eta_{\text {out }}(y)+O\left(\delta^{2}\right), \quad b(1)=D \mathrm{e}^{-3 \kappa / 2}+\delta \beta_{\text {out }}+O\left(\delta^{2}\right) .
$$

Unlike the top of the sheet, where the thickness and width are specified, here the perturbations $\eta_{\text {out }}$ and $\beta_{\text {out }}$ are to be found as part of the solution.

We scale into the boundary layer at $x=1$ by setting $x=1-\delta b_{0}(1) \bar{x}$ and $y=b_{0}(1) \bar{y}$, so that $(\bar{x}, \bar{y}) \in[0, \infty) \times[0,1]$. We write the outer solution given by (3.8) and (4.10) in inner variables and expand to get the matching conditions

$$
\begin{aligned}
& u \sim D+\delta\left[u_{1}(1)+D^{3} \mathrm{e}^{-3 \kappa}\left(\frac{\Gamma}{2}-\left(\kappa+\frac{\Gamma}{3}\right) \mathrm{e}^{\kappa / 2}\right) \bar{x}\right], \\
& v \sim-D^{3} \mathrm{e}^{-5 \kappa / 2}\left(\kappa+\frac{\Gamma}{3}\right) \frac{\bar{y}}{2}+\cdots, \\
& h \sim D^{-2} \mathrm{e}^{3 \kappa / 2}+\delta\left[A(1)+D^{-1 / 2} g(\bar{y})+\mathrm{e}^{-3 \kappa / 2}\left(-\Gamma+\left(\kappa+\frac{\Gamma}{3}\right) \mathrm{e}^{\kappa / 2}\right) \frac{\bar{x}}{2}\right], \\
& b \sim D \mathrm{e}^{-3 \kappa / 2}+\delta\left[b_{1}(1)+D^{3} \mathrm{e}^{-4 \kappa}\left(\kappa+\frac{\Gamma}{3}\right) \frac{\bar{x}}{2}\right]
\end{aligned}
$$




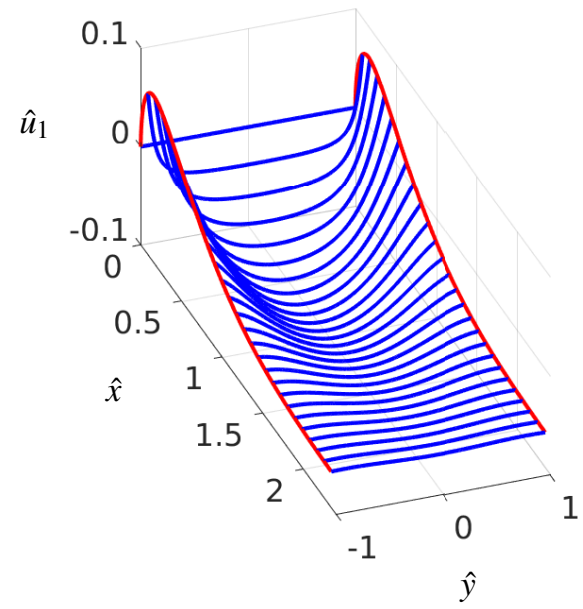

(a)

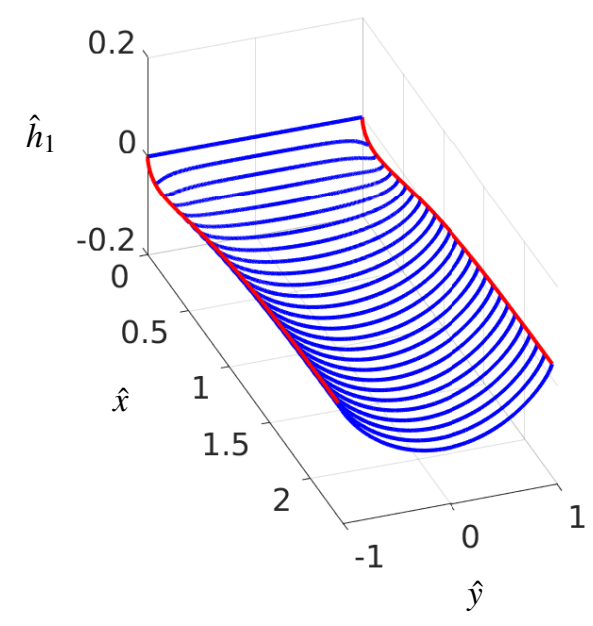

(c)

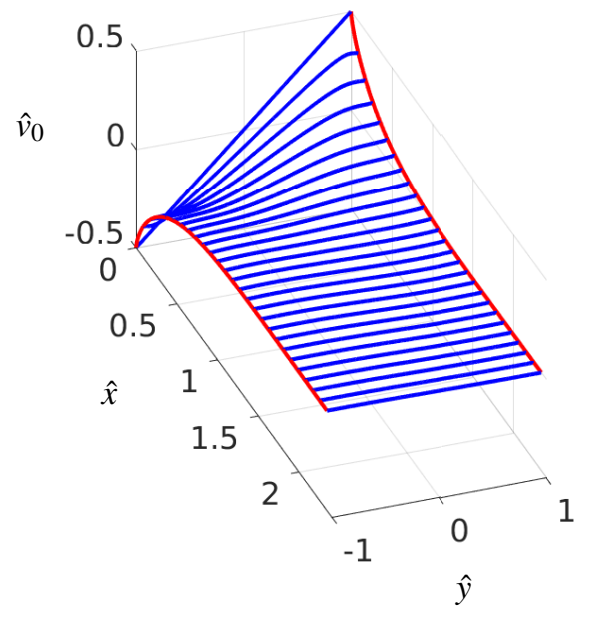

(b)

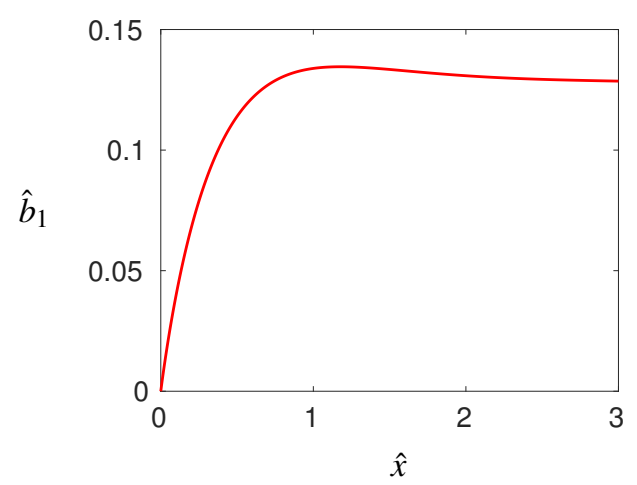

(d)

FIG. 7: Numerical solutions $\hat{u}_{1}(\hat{x}, \hat{y}), \hat{v}_{0}(\hat{x}, \hat{y}), \hat{h}_{1}(\hat{x}, \hat{y})$ and $\hat{b}_{1}(\hat{x})$ to the boundary-layer problem (5.3)(5.7). 


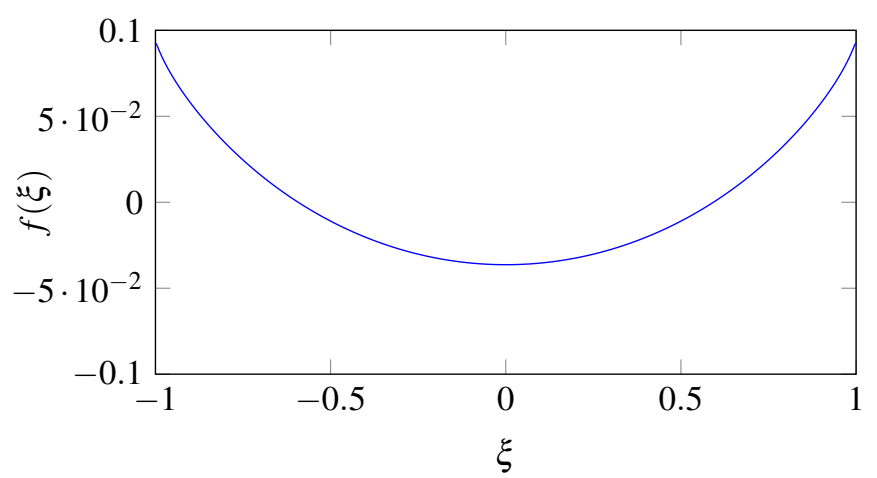

FIG. 8: Numerical solution for the function $f$ given by equation (5.11).

as $\bar{x} \rightarrow \infty$. These far-field conditions suggest the boundary layer scalings

$$
\begin{aligned}
& u=D+\delta D^{3} \mathrm{e}^{-5 \kappa / 2}\left[\left(\frac{\Gamma}{2} \mathrm{e}^{-\kappa / 2}-\left(\kappa+\frac{\Gamma}{3}\right)\right) \bar{x}-\left(\kappa+\frac{\Gamma}{3}\right) \bar{u}_{1}(\bar{x}, \bar{y})\right]+O\left(\delta^{2}\right) \\
& v=D^{3} \mathrm{e}^{-5 \kappa / 2}\left(\kappa+\frac{\Gamma}{3}\right)\left[-\frac{\bar{y}}{2}+\bar{v}_{0}(\bar{x}, \bar{y})\right]+O(\delta) \\
& h=D^{-2} \mathrm{e}^{3 \kappa / 2}+\delta \eta_{\text {out }}(y)+\delta \mathrm{e}^{-\kappa}\left[\left(-\Gamma \mathrm{e}^{-\kappa / 2}+\left(\kappa+\frac{\Gamma}{3}\right)\right) \frac{\bar{x}}{2}-\left(\kappa+\frac{\Gamma}{3}\right) \bar{h}_{1}(\bar{x}, \bar{y})\right]+O\left(\delta^{2}\right) \\
& b=D \mathrm{e}^{-3 \kappa / 2}+\delta \beta_{\text {out }}+\delta D^{3} \mathrm{e}^{-4 \kappa}\left(\kappa+\frac{\Gamma}{3}\right)\left[\frac{\bar{x}}{2}-\bar{b}_{1}(\bar{x}, \bar{y})\right]+O\left(\delta^{2}\right)
\end{aligned}
$$

We then find that the scaled variables $\bar{u}_{1}, \bar{v}_{0}, \bar{h}_{1}$ and $\bar{b}_{1}$ satisfy exactly the same partial differential equations and boundary conditions (5.3)-(5.7) as the corresponding variables $\hat{u}_{1}, \hat{v}_{0}, \hat{h}_{1}$ and $\hat{b}_{1}$ in the top boundary layer. We deduce that the barred functions are identical to the hatted functions and therefore satisfy the same far-field conditions (5.7). In particular, we must have $\bar{u}_{1}(\bar{x}, \bar{y}) \rightarrow c_{1}$ as $\bar{x} \rightarrow \infty$, where $c_{1}$ is again the numerical constant given by equation (5.8). The matching condition (5.14a) then leads to

$$
\frac{u_{1}(1)}{D}=-D^{2} \mathrm{e}^{-5 \kappa / 2}\left(\kappa+\frac{\Gamma}{3}\right) c_{1}
$$

where, from (4.10a), we have

$$
\frac{u_{1}(1)}{D}=\left(\kappa+\frac{\Gamma}{3}\right)\left(c_{1}-c_{2}+D \mathrm{e}^{-\kappa} c_{2}\right)+(\Gamma \alpha(1)-2 \kappa) d_{1} .
$$

The remaining integration constant $d_{1}$ is determined by equating the two expressions (5.16) and (5.17).

Similarly, the scaled sheet width must satisfy $\bar{b}_{1}(\bar{x}) \rightarrow c_{2}$ as $\bar{x} \rightarrow \infty$, where $c_{2}$ is given by equation (5.10). By substituting the expression $(5.15 \mathrm{~d})$ for $b$ into the matching condition $(5.14 \mathrm{~d})$, we obtain

$$
b_{1}(1)=\beta_{\text {out }}-D^{3} \mathrm{e}^{-4 \kappa}\left(\kappa+\frac{\Gamma}{3}\right) c_{2}=D^{2} \mathrm{e}^{-5 \kappa / 2}\left(\kappa+\frac{\Gamma}{3}\right) c_{2}+D \mathrm{e}^{-3 \kappa / 2}(\kappa+\Gamma \alpha(1)) d_{1} .
$$


We therefore determine explicitly how the output sheet width perturbation $\beta_{\text {out }}$ depends on the draw ratio $D$ and surface tension coefficient $\Gamma$, namely

$$
\beta_{\text {out }}=-D^{3} \mathrm{e}^{-3 \kappa}\left[A(1)-\left(c_{1}+c_{2}\right) \mathrm{e}^{-\kappa}\left(\kappa+\frac{\Gamma}{3}\right)\right],
$$

where $A(1)$ is given by (4.10c) and $\kappa$ satisfies (3.9).

Finally we match the sheet thickness across the bottom boundary layer by substituting the far-field behaviour (5.7b) into the matching conditions (5.14c) and (5.15c), resulting in

$$
\eta_{\text {out }}\left(b_{0}(1) \bar{y}\right)=A(1)+D^{-1 / 2} g(\bar{y})+\mathrm{e}^{-\kappa}\left(\kappa+\frac{\Gamma}{3}\right)\left[f(\bar{y})-c_{1}-c_{2}\right] .
$$

With $g$ given by $(5.12 \mathrm{c})$, we thus obtain the output sheet thickness profile in the form

$$
\eta_{\text {out }}\left(b_{0}(1) \bar{y}\right)=A(1)-\left(c_{1}+c_{2}\right) \mathrm{e}^{-\kappa}\left(\kappa+\frac{\Gamma}{3}\right)+D^{-1 / 2}\left[\eta_{\text {in }}(\bar{y})-\left(\kappa+\frac{\Gamma}{3}\right)\left(1+D^{1 / 2} \mathrm{e}^{-\kappa}\right) f(\bar{y})\right],
$$

with $A(1)$ again given by (4.10c). Note that the output thickness and width corrections satisfy the identity

$$
h_{0}(1) \beta_{\text {out }}+b_{0}(1) \int_{0}^{1} \eta_{\text {out }}(\bar{y}) \mathrm{d} \bar{y} \equiv 0,
$$

as a consequence of net mass conservation.

\section{Results}

\subsection{Dependence on parameters}

We now investigate the influence of the draw ratio $D$ and surface tension parameter $\Gamma$ on the final width and thickness of the redrawn glass sheet. Although these are in principle determined by the analytical formulae (5.19) and (5.21), the qualitative trends are somewhat obscured by the fact that these functions are lengthy and depend the parameter $\kappa$, which in turn depends implicitly on $D$ and $\Gamma$ through the expression (3.9). For the moment we focus on the drawing of an initially uniform preform, and therefore set $\eta_{\text {in }} \equiv 0$.

In figure 9 we plot the correction to the final width, $\beta_{\text {out }}$, as a function of the draw ratio $D$. For small to moderate values of the surface tension parameter $\Gamma$, the width correction $\beta_{\text {out }}$ is positive for all values of the draw ratio $D$. However, if $\Gamma$ exceeds a critical value

$$
\Gamma^{*}=\frac{6}{c_{2}-c_{1}}\left(2 c_{2}+c_{1}+\sqrt{\left(4 c_{2}-c_{1}\right)\left(2 c_{2}+c_{1}\right)}\right) \approx 16.46,
$$

then the width correction is negative at small draw ratios, so the effect of the finite heater zone is to make the sheet slightly narrower than expected. In the limit of very high draw ratio, the width correction tends to zero like $\beta_{\text {out }} \sim\left(c_{2}+c_{1} / 2\right) D^{-1 / 2} \log (D)$. Therefore $\beta_{\text {out }}$ always takes its maximum value at some intermediate value of $D$. The size of the relative width correction $\beta_{\text {out }} / b_{0}(1)$ again may be negative at small draw ratios if $\Gamma$ is sufficiently large. However, $\beta_{\text {out }} / b_{0}(1)$ ultimately becomes an increasing function of $D$, with $\beta_{\text {out }} / b_{0}(1) \sim\left(c_{2}+c_{1} / 2\right) \log (D)$ as $D \rightarrow \infty$, so that the relative effect of the finite heater zone is larger for larger draw ratios (see figure $9 \mathrm{~b}$ ). 


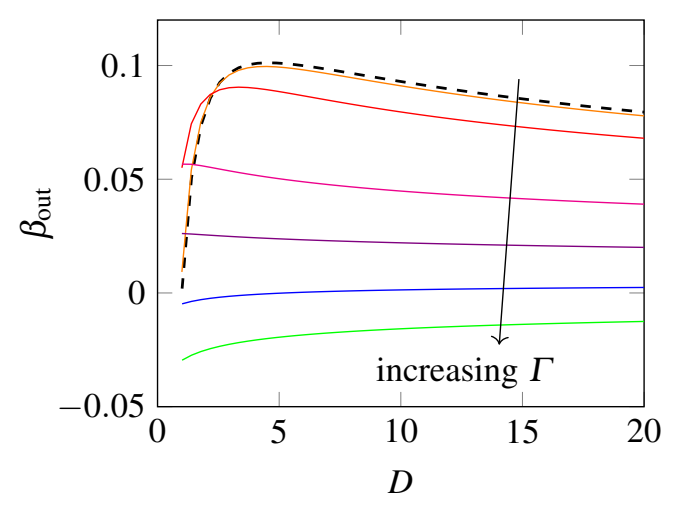

(a) Correction to final sheet width.

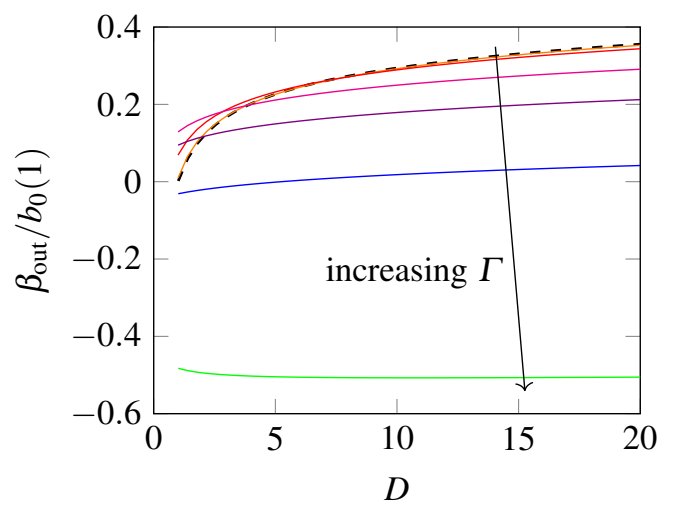

(b) Normalized width correction.

FIG. 9: Correction $\beta_{\text {out }}$ to final sheet width, given by (5.19), as a function of draw ratio $D$ for surface tension parameter $\Gamma=0.0$ (black dashed), 0.1 (orange), 1.0 (red), 5.0 (magenta), 10.0 (violet), 20.0 (blue) and 50.0 (green).

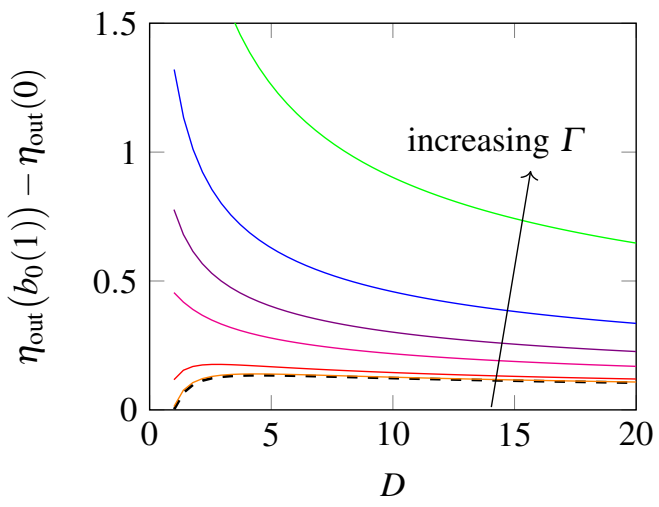

(a) Variation in thickness correction.

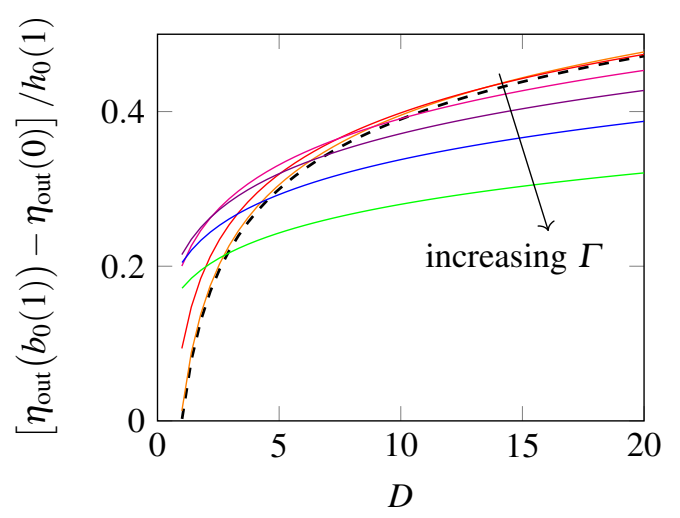

(b) Normalized thickness variation.

FIG. 10: Difference between edge and centre ouput sheet thickness as a function of draw ratio $D$ for surface tension parameter $\Gamma=0.0$ (black dashed), 0.1 (orange), 1.0 (red), 5.0 (magenta), 10.0 (violet), 20.0 (blue) and 50.0 (green). 
The calculations carried out in $\S 4$ demonstrate not only that the aspect ratio of the sheet changes by an $O(\delta)$ amount due to the finite length of the heater zone, but also that the final thickness profile becomes non-uniform at this order. To quantify the induced thickness variations across the sheet, in figure 10a we plot the difference

$$
\eta_{\text {out }}\left(b_{0}(1)\right)-\eta_{\text {out }}(0)=(f(1)-f(0))\left(D^{-1 / 2}+\mathrm{e}^{-\kappa}\right)\left(\kappa+\frac{\Gamma}{3}\right)
$$

between the edge and centre values of the output sheet thickness versus the draw ratio. The thickness variation attains a local maximum at some critical draw ratio $D$ when the surface tension parameter $\Gamma \lesssim 4.02$, but ultimately decreases for large draw ratios, regardless of the size of $\Gamma$. The thickness variation increases as the surface tension parameter $\Gamma$ increases, indicating that non-uniformities in the final sheet thickness are exacerbated by the presence of surface tension, which acts to increase necking-in of the sheet. However, the relative thickness variation across the sheet width, compared to the leading-order thickness, increases as the draw ratio $D$ increases (see figure 10b), and is maximised at a critical value of surface tension parameter $\Gamma$ (see figure 11), beyond which it decreases as $\Gamma$ increases.

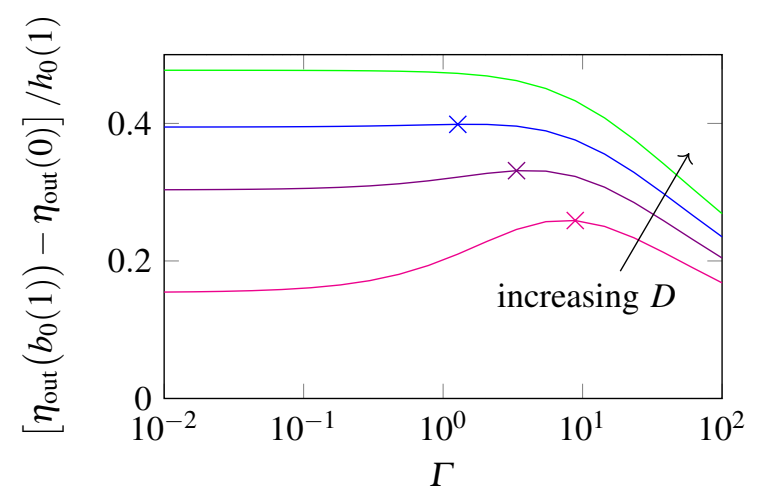

FIG. 11: Relative variation in ouput sheet thickness as a function of the surface tension parameter $\Gamma$, with draw ratios $D=2.0$ (magenta), 5.0 (violet), 10.0 (blue) and 20.0 (green). Local maxima are marked with crosses.

We emphasise that, even though the absolute thickness variation is increased by surface tension, the size of this variation relative to the leading-order thickness actually decreases for sufficiently large $\Gamma$, because the leading-order thickness is also increased by the presence of surface tension. This suggests a possible procedure for reducing thickness variations while keeping the heater zone length fixed: instead of using a preform whose aspect ratio is exactly the desired final aspect ratio, use a preform that is slightly thinner, and increase the temperature to decrease the viscosity $\mu$ and increase the effect of surface tension $\Gamma$. Surface tension will act to alter the aspect ratio in the leading-order solution (3.8) as desired, while the relative thickness variations will be smaller than in a sheet drawn without surface tension.

\subsection{Comparison with numerical experiments}

We now test the validity of our model by comparing our predictions with numerical solutions to the three-dimensional Stokes equations with appropriate boundary conditions obtained using the finite- 
element package Polyflow (Ansys Inc. Polyflow, 2013). Uniform viscosity was prescribed throughout the sheet, and solutions were calculated with a fixed thin-sheet parameter $\varepsilon=0.05 \ll 1$ and a range of values of the geometrical parameter $\delta$, surface tension parameter $\Gamma$ and draw ratio $D$.

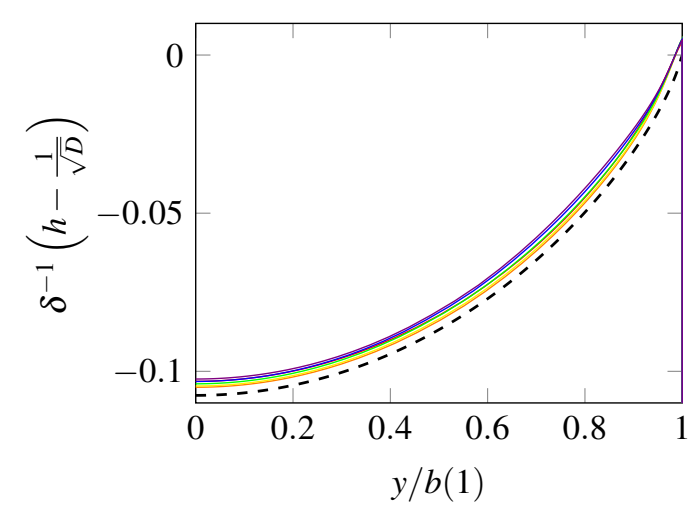

(a)

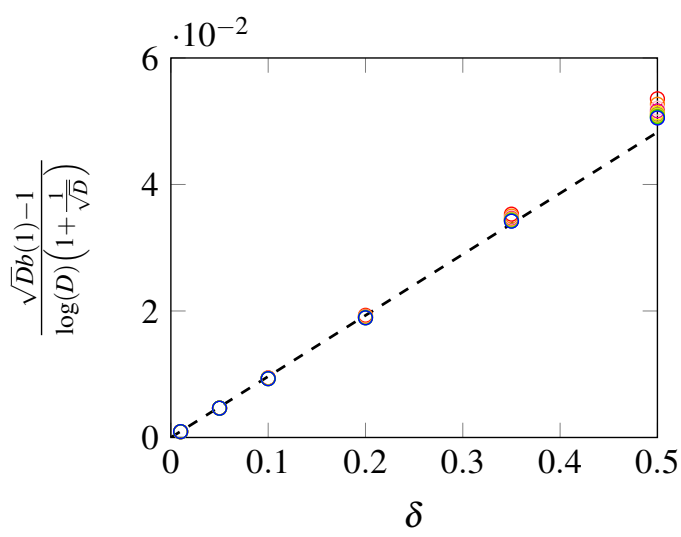

(b)

FIG. 12: Scaled difference in thickness and width from leading-order prediction in the absence of surface tension $(\Gamma=0)$. The coloured lines/circles show numerical solutions to the three-dimensional Stokes equations computed in Polyflow with $\delta=0.05$, and the black-dashed lines are asymptotic predictions. (a) Scaled thickness profile for $\delta=0.01$ (red), 0.05 (orange), 0.1 (yellow), 0.2 (green), 0.35 (blue) and 0.5 (violet) with draw ratio $D=20$, and the asymptotic solution in the limit $\delta \rightarrow 0$ (6.3b). (b) Scaled width as a function of $\delta$ for $D=5$ (red), $D=10$ (orange), $D=15$ (pink), $D=20$ (magenta), $D=25$ (yellow), $D=30$, (brown), $D=35$ (lime), $D=40$ (olive), $D=45$ (green), and $D=50$ (blue), and the asymptotic prediction (6.3a) for the change in width in the limit $\delta \rightarrow 0$.

We first consider sheets redrawn at draw ratio $D=20$ in the absence of surface tension. With $\Gamma=0$, the expressions (5.19) and (5.21) for the output width and thickness perturbations simplify to

$$
\begin{gathered}
\frac{b(1)-D^{-1 / 2}}{\delta} \sim \beta_{\text {out }}=\frac{\log (D)}{\sqrt{D}}\left(1+\frac{1}{\sqrt{D}}\right)\left(c_{2}+\frac{c_{1}}{2}\right), \\
\frac{h(1, y)-D^{-1 / 2}}{\delta} \sim \eta_{\text {out }}(y)=\frac{\log (D)}{\sqrt{D}}\left(1+\frac{1}{\sqrt{D}}\right)\left(-c_{2}-\frac{c_{1}}{2}+f\left(y / b_{0}(1)\right)\right) .
\end{gathered}
$$

In figure 12a we plot the left-hand side of (6.3b) as computed in Polyflow using small but nonzero values of $\delta$. The coloured lines are the same data as in figure 2, but the width and thickness of the sheet are scaled so that the profiles all collapse onto one curve. A black dashed line shows the thickness correction (6.3b) predicted by the asymptotic solution. The asymptotic solution captures the thickness variations extremely well, even for values of $\delta$ as large as 0.5 and also for values small enough to be comparable to the thin-sheet parameter $\varepsilon$.

The change in width as a function of $\delta$ is plotted in figure $12 \mathrm{~b}$, and compared with numerical solutions to the three-dimensional Stokes equations for a range of draw ratios $D$. The data are scaled to collapse onto one curve for all values of $D$. The asymptotic prediction (6.3a) is shown as a black dashed line. The agreement between the asymptotics and full numerical solutions is excellent, and evidently becomes increasingly accurate as $\delta \rightarrow 0$, as expected. 
In figure 13, we show the final thickness profile of sheets drawn with surface tension parameter $\Gamma=1$, for two values of the aspect ratio $\delta=0.05$ and $\delta=0.2$. The data are scaled with $1 / \sqrt{D}$ in both directions so that the scaled width and average thickness would both be 1 in the absence of surface tension. The agreement between the full numerical solution and the two-term asymptotic solution (5.13) is good outside a region of size $O(\varepsilon \delta)$ at the edge of the sheet, where surface tension smooths out the sharp corners. This local smoothing is not captured by our model, and gives rise to a small offset in the predicted width compared to the actual width, which persists over different values of $\delta$.
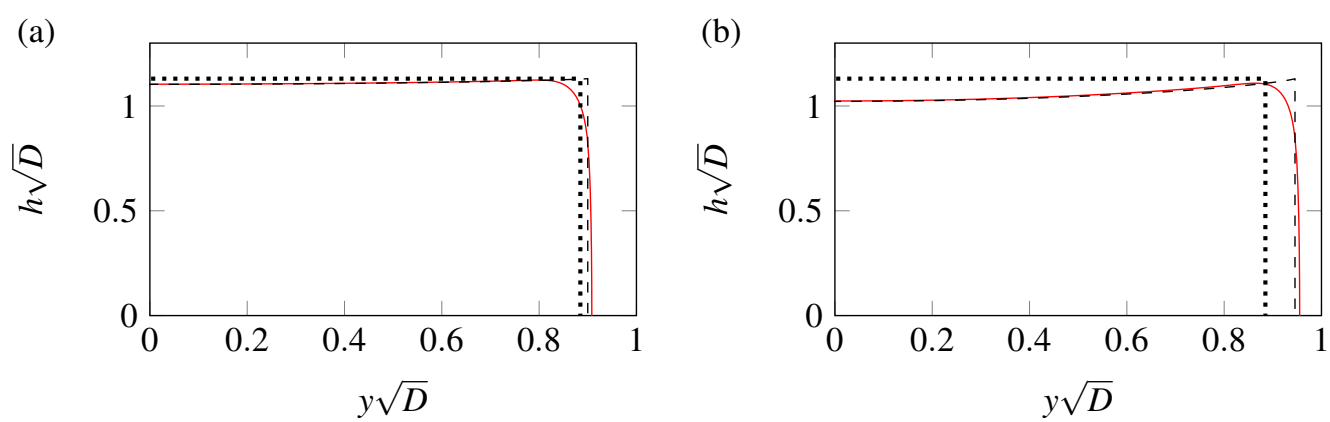

FIG. 13: Final thickness profile of sheets drawn at draw ratio $D=20$ with (a) $\delta=0.05$ and (b) $\delta=0.2$ and surface tension $\Gamma=1$. Numerical solutions to the full three-dimensional problem computed in Polyflow are shown in red, the black dotted lines show the leading-order asymptotic solution (3.8) in the limit $\delta \rightarrow 0$, and the black dashed lines show the two-term asymptotic solution (5.13).

\subsection{Inverse problem}

Now we return to the inverse problem of modifying the preform shape to control the output thickness profile. In particular, we would like to choose the input thickness perturbation $\eta_{\text {in }}(y)$ to ensure that the final product is uniform, i.e. that $\eta_{\text {out }}(y)$ is constant. From equation (5.21), we see that this is easily achieved by setting

$$
\eta_{\text {in }}(y)=\left(\kappa+\frac{\Gamma}{3}\right)\left(1+D^{1 / 2} \mathrm{e}^{-\kappa}\right) f(y),
$$

where $f$ is the numerically determined function plotted in figure 8. As shown in O'Kiely (2017), it is also possible to arrive at the optimal preform shape (6.4) by the method employed for a short heater zone in O' Kiely et al. (2015), i.e., by introducing a "compressive" draw ratio $D^{*}=1 / D$ and rescaling the variables appropriately. Comparing the solutions (5.21) and (6.4) to the forward and inverse problems, we see that, from a practical point of view, a straightforward procedure can be followed, which works for all values of the surface tension parameter $\Gamma$. Upon observing some thickness variation in a redrawn sheet: (i) determine the thickness variation from the mean across the width and (ii) remove the same profile from the preform, scaled with a factor $\sqrt{D}$ and stretched across the preform width.

\section{Discussion}

In this paper, we analyse the glass redraw process in the limit where the heater zone is long compared to the width of the preform, and investigate how thickness variations across the width of the final product 
are affected by the length of the heater zone. We derive a mathematical model to predict how the thickness profile and width of the final product depend on the process parameters. We first address the problem at leading order in the small aspect ratio of width to length, and find that the preform shape is preserved at leading order in the absence of surface tension, with necking-in of the sheet edges increasing as the importance of surface tension relative to viscous effects increases.

By matching between the bulk of the sheet and boundary layers at either end of the heater zone where the solution adjusts rapidly to satisfy the boundary conditions, we determine that the correction terms for a sheet of finite length are of $O(\delta)$, with $\delta=$ preform half-width/heater zone length. Schultz \& Davis (1982) have previously bypassed the solution of the boundary-layer problem for a fibre by applying an averaged version of the boundary conditions directly to the bulk solution, but this approach fails to predict the existence of $O(\delta)$ terms, instead giving correction terms of $O\left(\delta^{2}\right)$. The $O(\delta)$ corrections can be determined by solving the two-dimensional boundary-layer problems at the sheet ends numerically. We find that the governing equations in the two boundary layers are equivalent, and can be rescaled to be independent of the draw ratio and rescaled capillary number, so that the boundary-layer problem needs only to be solved once.

We compare our asymptotic results with numerical solutions to the three-dimensional Stokes equations. In the absence of surface tension, the agreement is excellent and the scalings predicted by the asymptotics allow all the numerical data to be collapsed onto one curve for any value of $\delta$ and the draw ratio $D$. When surface tension is included, the agreement between numerics and asymptotics is good except for small regions near the corners of the sheet, which are smoothed out by surface tension. Our model relies on a thin-sheet approximation that breaks down in the region at the edge of the sheet where the smoothing effect occurs, and so smoothing can only be resolved by solving the full Stokes equations inside this edge region.

We use the two-term asymptotic solution to the long-heater-zone problem to investigate how the geometric properties of the final product depend on the process parameters. The size of the higher-order corrections relative to the leading-order solution increases as the draw ratio $D$ increases (except when $\Gamma$ is large and the correction changes sign). The width correction is typically positive, except in the limit where surface tension dominates over drawing, in which case it may be negative. The relative thickness variation across the sheet width is maximized at a critical value of the surface tension parameter, beyond which it decreases as the effect of surface tension increases. It may therefore be advantageous to increase the effect of surface tension (e.g. by increasing the furnace temperature) to decrease thickness variations, even though the aspect ratio of thickness to width will be altered, since it is more straightforward to alter the aspect ratio of the preform than it is to modify the initial thickness profile.

We restrict our attention to sheets with uniform viscosity in this paper. For spatially varying viscosity, we still anticipate boundary layers of size $O(\delta)$ near the inlet and outlet where the solution adjusts to satisfy the boundary conditions, giving rise to corrections of size $O(\delta)$ to the leading-order solution. In many industrial processes, the viscosity varies principally in the direction of drawing, so that $\mu=\mu(x)$ to a first approximation. In such a case, the viscosity variations may be eliminated from the problem by transforming to the new axial variable $X=\int \mathrm{d} x / \mu(x)$, and the results of this paper may thus be applied directly, so even when the temperature and viscosity adjust as the sheet is heated or cooled, the same boundary layer structure with $O(\delta)$ corrections exists (see O'Kiely, 2017, for further discussion). We have also neglected gravity in our study; this can easily be taken into account by including an extra term in the momentum equations.

Finally, we address the inverse problem of determining the optimal preform shape that will redraw to a sheet of uniform thickness. Similarly to the short-heater-zone case (O'Kiely et al., 2015), it is possible to pose this inverse problem in terms of the forward problem. In the long-heater-zone case studied 
here, the inverse problem may be solved in the same computation as the forward problem. Furthermore, we identify a practical solution which involves simply measuring the thickness variation in a product redrawn from a rectangular preform, and applying a straightforward scaling to determine the preform modification required, with no computation necessary.

This paper, together with the numerical simulations of Filippov \& Zheng (2010) and the shortheater-zone analysis carried out in O'Kiely et al. (2015), demonstrates that thick edges are an endemic problem in the redraw of thin glass sheets. A preform with uniform thickness will always redraw to a product that is thicker at the edge than in the centre, regardless of the length of the heater zone. However, our analysis allows the variations in the glass sheet shape to be predicted and controlled with high accuracy, facilitating further progress in the manufacture of products with particular desired aspect ratios and thicknesses.

\section{Acknowledgement}

DOK is grateful for funding from Schott AG and the Mathematical Institute, University of Oxford. IMG gratefully acknowledges support from the Royal Society through a University Research Fellowship. This paper benefited from extremely helpful suggestions provided by Professor John Lister.

\section{REFERENCES}

Ansys Inc. Polyflow (2013) Release 15.0. .

Burke, S. (2016, (accessed 22 March 2016)) This glass can bend in half without shattering (online video clip). http://edition.cnn.com/videos/cnnmoney/2016/03/18/ bendable-glass-schott-burke-pkg.cnn-money/video/playlists/technology/. CNN Money.

d'Halewyu, S., Agassant, J. F. \& Demay, Y. (1990) Numerical simulation of the cast film process. Polym. Eng. Sci., 30(6), 335-340.

Dobroth, T. \& Erwin, L. (1986) Causes of edge beads in cast films. Polym. Eng. Sci., 26(7), 462-467.

Filippov, A. \& Zheng, Z. (2010) Dynamics and shape instability of thin viscous sheets. Phys. Fluids, 22(2), 023601. Horvatitsch, T. (2016) Ultra-thin glass. Schott Solutions 1/2016, pages 6-11.

Howell, P. D. (1994) Extensional thin layer flows. PhD thesis, University of Oxford.

Howell, P. D. (1996) Models for thin viscous sheets. Eur. J. Appl. Math., 7(04), 321-343.

Matovich, M. A. \& Pearson, J. R. A. (1969) Spinning a molten threadline. Steady-state isothermal viscous flows. Ind. Eng. Chem. Fund., 8(3), 512-520.

O'Kiely, D. (2017) Mathematical models for the glass sheet redraw process. $\mathrm{PhD}$ thesis, University of Oxford.

O'Kiely, D., Breward, C. J. W., Griffiths, I. M., Howell, P. D. \& Lange, U. (2015) Edge behaviour in the glass sheet redraw process. J. Fluid Mech., 785, 248-269.

Schultz, W. W. \& Davis, S. H. (1982) One-dimensional liquid fibers. J. Rheol., 26(4), 331-345.

Silagy, D., Demay, Y. \& Agassant, J. F. (1999) Numerical simulation of the film casting process. Int. J. Num. Meth. $F l$, 30(1), 1-18.

Silliman, W. \& Scriven, L. (1980) Separating flow near a static contact line: Slip at a wall and shape of a free surface. J. Comput. Phys., 34(3), 287-313.

Trouton, F. T. (1906) On the coefficient of viscous traction and its relation to that of viscosity. P. Roy. Soc. Lond. A Mat., 77(519), 426-440. 